\title{
Limiting factors of current thoracic aortic endovascular technologies
}

\author{
Thomas G. Gleason \\ Division of Cardiac Surgery, Department of Surgery, University of Maryland School of Medicine, Baltimore, MD, USA \\ Correspondence to: Thomas G. Gleason, MD. Division of Cardiac Surgery, Department of Surgery, University of Maryland School of Medicine, 110 S. \\ Paca St., 7th Floor, Baltimore, MD 21201, USA. Email: tgleason@som.maryland.edu.
}

Submitted Mar 05, 2021. Accepted for publication Jun 16, 2021.

doi: 10.21037/acs-2021-taes-10

View this article at: https://dx.doi.org/10.21037/acs-2021-taes-10

It has been over thirty years since Volodos et al. and Parodi et al. reported separately on the early experiences with their archetypal stent grafting devices used for treating aneurysmal aortic disease $(1,2)$. Dake et al.'s seminal manuscript detailing what is now known as thoracic endovascular aortic repair (TEVAR), set the stage for what has amounted to a revolution in the management of aortic disease (3). TEVAR is now the preferred method of treatment for many thoracic aortic diseases by both physicians and patients, largely predicated on the minimized immediate impact of the surgical procedure itself. Despite the obvious attractiveness of the approach, endovascular therapies, and TEVAR specifically, continue to have major deficiencies that limit both their use and efficacy.

Deficits persist in device delivery size/caliber, maneuverability, conformability, branch vessel- or biocompatibility, and compliance mismatch. Though there are now many United States Food and Drug Administration (FDA) approved stent grafts for use in the descending thoracic aorta, they all have limitations inherent to their design, versatility, and impact. To date, there are no FDA approved stent grafts available for the ascending aorta, aortic arch or thoracoabdominal aorta. In each of these areas, there are devices in use under industry-sponsored or physician-sponsored Investigational Device Exemption (IDE) clinical trials, but the experience with them and the applicability of their use are quite limited.

Despite marked reductions in delivery system caliber across all of the newer generation FDA approved devices, the currently available systems still require relatively large arterial blood vessels for access (approximately 6 to $6.5 \mathrm{~mm}$ or greater) which limit their application in patients with smaller or more diseased access arteries (e.g., femoral).
Similarly, current delivery systems are neither steerable nor articulate despite being bendable. Consequently, inevitable, even requisite, endoluminal vessel wall-scrape and/or pressure injuries with the potential for plaque, thrombus and/or debris dislodgement remain a regular phenomenon that can give rise to cerebrovascular accidents caused by embolic phenomena, aortic dissection, or even disruption, particularly in the circumstance of a torturous thoracoabdominal aorta or a steeply angulated aortic arch. Better, steerable and even articulating delivery systems are actively in development in the endovascular research space, and it is likely that such developments will ultimately improve maneuverability.

The lack of optimal conformability remains a limitation of current thoracic aortic stent grafts with short, often steeply angulated landing zones in the aortic arch. Consequent seal-zone capabilities can be hindered by nonuniform or inadequately distributed radial force that renders incomplete stent graft-to-aortic wall apposition with current stent designs (e.g., "bird's beaking") that invokes type 1 endoleaking, graft infolding and/or may contribute to the development of retrograde type A aortic dissection (4). Similarly, branched and fenestrated endografts that have been designed for the thoracoabdominal aorta are limited by the markedly different branch vessel anatomy seen across the spectrum of human disease and by the consequent difficulty in achieving adequate seals at branch sites. Recognition of inadequate conformability or apposition and the difficulty of accommodating branch vessel anatomic variability is a harbinger for both delayed complications or the need for secondary reinterventions (5). The evolution of branched stent grafts will likely broaden the applicability of TEVAR in this regard and allow endovascular treatment of 
more proximal thoracic aortic disease, thus mitigating some of the conformability limitations of non-branched stent grafts. However, currently available branched stent grafts remain severely limited in their anatomic suitability for most forms of aortic arch disease (6). Alternatively, utilizing three dimensional-printed, customized stent graft formulations and leveraging computational modeling techniques (e.g., fluid-structure interaction) may provide a means for "custom fit" stent grafts that could further mitigate and potentially alleviate many conformational limitations $(7,8)$.

Inflammatory responses, infection susceptibility and biocompatibility inadequacies also persist portending to post-implantation syndromes and stent graft infections that, while rare, can be both debilitating and even lethal. Simple antibiotic graft soaking has not been demonstrated to reduce prosthetic infection risk, likely because systemic clearance of even dried antibiotic on graft material occurs so rapidly following implantation. Sustained-release antibioticimpregnated device technologies are being developed but have as yet unproven efficacy. Alternatively, tissueengineered technologies that incorporate human cells and subsequent matrix deposition within a biodegradable scaffold may ultimately be feasible and offer a better biocompatibility and infection resistance than non-biodegradable materials like polyester or polytetrafluoroethylene (9).

Perhaps the most impactful unmet element of endovascular stent graft development and innovation that needs more pointed attention relates to compliance mismatch. The importance of the Windkessel effect (aortic wall compliance functions as an elastic reservoir storing energy during systole and releasing it during diastole-analogous to a capacitor) and the inherent intrinsic aortic elastic properties on both cardiac function and cardiovascular health has not been accounted for in current aortic graft or stent graft material designs. The Windkessel effect is dramatically reduced or even lost with the implantation of these prosthetic grafts (10). The consequence of the resultant compliance mismatch and loss of the Windkessel effect that occurs with endovascular stent grafting is poorly characterized but likely manifests at a minimum as left ventricular hypertrophy that teleologically will have a negative impact on long-term survival.

In summary, there is little argument that stent grafts have had a remarkable impact on thoracic aortic disease and surgical practice. As patients and clinicians continue to seek and desire non- or less invasive therapies for diseases that would otherwise require life-threatening procedures, that in and of themselves invoke disease and impact both short- and long-term survival, there is a critical need to make further strides toward broadening the applicability, improving the durability, and limiting the morbidity of these endovascular technologies.

\section{Acknowledgments}

Funding: None.

\section{Footnote}

Conflicts of Interest: Dr. TGG serves on a Medical Advisory Board for Abbott without remuneration.

Open Access Statement: This is an Open Access article distributed in accordance with the Creative Commons Attribution-NonCommercial-NoDerivs 4.0 International License (CC BY-NC-ND 4.0), which permits the noncommercial replication and distribution of the article with the strict proviso that no changes or edits are made and the original work is properly cited (including links to both the formal publication through the relevant DOI and the license). See: https://creativecommons.org/licenses/by-nc-nd/4.0/.

\section{References}

1. Parodi JC, Palmaz JC, Barone HD. Transfemoral intraluminal graft implantation for abdominal aortic aneurysms. Ann Vasc Surg 1991;5:491-9.

2. Volodos NL, Karpovich IP, Troyan VI, et al. Clinical experience of the use of self-fixing synthetic prostheses for remote endoprosthetics of the thoracic and the abdominal aorta and iliac arteries through the femoral artery and as intraoperative endoprosthesis for aorta reconstruction. Vasa Suppl 1991;33:93-5.

3. Dake MD, Miller DC, Semba CP, et al. Transluminal placement of endovascular stent-grafts for the treatment of descending thoracic aortic aneurysms. N Engl J Med 1994;331:1729-34.

4. Marrocco-Trischitta MM, Spampinato B, Mazzeo G, et al. Impact of the Bird-Beak Configuration on Postoperative Outcome After Thoracic Endovascular Aortic Repair: A Meta-analysis. J Endovasc Ther 2019;26:771-8.

5. Belvroy VM, Romarowski RM, van Bakel TMJ, et al. Impact of Aortic Tortuosity on Displacement Forces in Descending Thoracic Aortic Aneurysms. Eur J Vasc Endovasc Surg 2020;59:557-64.

6. Fujimura N, Ichihashi S, Motoki M, et al. Anatomical 
Analysis and Feasibility Study of Next-Generation Fenestrated or Branched Stent-Grafts for the Treatment of Arch Aneurysms. J Endovasc Ther 2020;27:777-84.

7. Tang F, Hu C, Huang S, et al. An Innovative Customized Stent Graft Manufacture System Assisted by ThreeDimensional Printing Technology. Ann Thorac Surg 2021;112:308-14.

8. Jayendiran R, Nour B, Ruimi A. Fluid-structure interaction (FSI) analysis of stent-graft for aortic endovascular aneurysm repair (EVAR): Material and structural considerations. J Mech Behav Biomed Mater

Cite this article as: Gleason TG. Limiting factors of current thoracic aortic endovascular technologies. Ann Cardiothorac Surg 2021;10(6):787-789. doi: 10.21037/acs-2021-taes-10
2018;87:95-110.

9. Kawajiri H, Mizuno T, Moriwaki T, et al. Development of tissue-engineered self-expandable aortic stent grafts (Bio stent grafts) using in-body tissue architecture technology in beagles. J Biomed Mater Res B Appl Biomater 2015;103:381-6.

10. Dobson G, Flewitt J, Tyberg JV, et al. Endografting of the descending thoracic aorta increases ascending aortic input impedance and attenuates pressure transmission in dogs. Eur J Vasc Endovasc Surg 2006;32:129-35. 